\title{
QCD sum rules and neutron-proton mass difference
}

\author{
Kwei-Chou Yang and W-Y. P. Hwang \\ Department of Physics, National Taiwan University, Taipei, Taiwan 10764, Republic of China \\ E. M. Henley \\ Department of Physics FM-15, University of Washington, Seattle, Washington 98195 \\ L. S. Kisslinger \\ Department of Physics, Carnegie-Mellon University, Pittsburgh, Pennsylvania 15213
}

(Received 24 August 1992)

\begin{abstract}
We use the method of QCD sum rules to investigate the neutron-proton mass difference. We include diagrams consistently up to dimension 9, assuming different up and down current-quark masses $\left(m_{u} \neq m_{d}\right)$, and distinguish between $\langle 0|: \bar{u} u:| 0\rangle$ and $\langle 0|: \bar{d} d:| 0\rangle$, the condensates of the up and down quarks. Using the typical current-quark masses $m_{u}=5.1 \mathrm{MeV}$ and $m_{d}=8.9 \mathrm{MeV}$ and the standard condensate values for average current-quark masses, we perform numerical analyses of the resultant QCD $\not p$ and unity sum rules. In particular, numerical analyses of the difference equation from the $\not p$ sum rules yield $M_{n}-M_{p}=(1.35 \pm 0.24) \mathrm{MeV}$ or $(1.42 \pm 0.19) \mathrm{MeV}$, depending on the method of the analysis. Analogously, the difference equation from the unity sum rules yields $M_{n}-M_{p}=(1.35 \pm 0.35) \mathrm{MeV}$ or $(0.95 \pm 0.25) \mathrm{MeV}$. These predictions are consistent among themselves and all are in reasonable agreement with the experimental value of $1.29 \mathrm{MeV}$.
\end{abstract}

PACS number(s): 12.70. $+\mathrm{q}, 11.50 . \mathrm{Li}, 12.38 . \mathrm{Lg}, 14.20 . \mathrm{Dh}$

\section{INTRODUCTION}

Quantum chromodynamics (QCD), and SU(3) nonAbelian gauge theory, is the presently accepted theory of strong interactions among quarks and gluons. Although QCD has defied attempts to obtain solutions to strong interaction problems, it has been conjectured that the ground state, or the vacuum $|0\rangle$, possesses nontrivial structures such as nonzero gluon and quark condensates, $\left\langle 0\left|: G_{\mu \nu}^{a} G^{a \mu \nu}:\right| 0\right\rangle \neq 0$ and $\langle 0|: \bar{q} q:| 0\rangle \neq 0$. Although it is highly desirable to use, e.g., numerical simulations with lattice QCD to substantiate any nontrivial structure associated with the QCD vacuum, it is nevertheless useful to tackle problems in strong interaction physics with models which hew as close as possible to QCD. This has led Shifman, Vainshtein, and Zakharov [1] to propose the method of QCD sum rules. In short, the method of QCD sum rules is built upon the working hypothesis that there is a kinematic region, say of momentum transfer squared $Q^{2}$ in the range of a couple of $\mathrm{GeV}^{2}$, in which Green's functions (or correlation functions) may be evaluated at the quark-gluon level, using perturbative QCD augmented with nonperturbative gluon and quark condensates to replace large-distance physics; these results are compared with those obtained from dispersion relations at the hadronic level. The method yields predictions on the physical parameters, such as masses, magnetic moments, and axial-vector couplings, associated with low-lying baryons and mesons.

Treating both the $u$ and $d$ quarks as massless, Ioffe [2] and, independently, Chung et al. [3] obtained QCD sum rules for baryon masses. Later, Belyaev and Ioffe [4] improved the results by keeping terms up to dimension 9 in the derivation. As isospin symmetry breaking is yet to be taken into account explicitly, these sum rules cannot be used directly to investigate the neutron-proton mass difference, which requires consistent inclusion of the effects due to $m_{d} \neq m_{u}$ and $\langle 0|: \bar{u} u:| 0\rangle \neq\langle 0|: \bar{d} d:| 0\rangle$. Nevertheless, Hatsuda, Hogaasen, and Prakash [5] have discussed the neutron-proton mass difference, using the mass sum rules of $\Sigma$ and $\Xi$, obtained previously by Reinders, Rubinstein, and Yazaki [6], and making a simple substitution $m_{s} \rightarrow m_{d}$. The purpose of the present paper is to take up again the problem of the neutron-proton mass difference by keeping diagrams consistently up to dimension 9 and distinguishing between $\langle 0|: \bar{u} u:| 0\rangle$ and $\langle 0|: \bar{d} d:| 0\rangle$. The contributions of excited states and the continuum are taken into account through a standard ansatz $[1,7]$. At the hadron level, we use a threshold in the form of a $\theta$ function to approximate the contributions from the excited states and the continuum [1,2,7]-in particular, a threshold energy $W$ is chosen such that, for $s>W^{2}$, the cut in the spectral function (which enters the dispersion relation at the hadron level) is identified with that obtained at the quark level. We include a discussion of the difference of $W_{n}$ and $W_{p}$.

The rest of this paper is organized as follows. In Sec. II, we derive the QCD sum rules, which allow an investigation of the neutron-proton mass difference to be made. In Sec. III, typical numerical results are described and discussed. Section IV contains a brief summary.

\section{THE METHOD}

The method of QCD sum rules consists of the evaluation of a correlation function (or Green's function) both 
at the quark-gluon level and at the hadron level and subsequent identification of the results with each other in a certain range of momentum transfer squared $Q^{2}$. For studying proton and neutron masses, we may consider the correlation function specified by

$$
\Pi(p)=i \int d^{4} x e^{i p x}\langle 0|T[\eta(x) \bar{\eta}(0)]| 0\rangle,
$$

where for the nucleon current $\eta(x)$ we use a standard (but not unique) form $[2,8]$

$$
\eta(x)=\epsilon^{a b c}\left[u^{a T}(x) C \gamma_{\mu} u^{b}(x)\right] \gamma^{\mu} \gamma^{5} d^{c}(x),
$$

with $a, b$, and $c$ color indices and $C=-C^{T}$ the charge conjugate operator. The current $\eta(x)$ is chosen such that

$$
\begin{aligned}
& \left\langle 0|\eta(0)| \frac{1}{2}^{+}\right\rangle=\lambda^{+} v(p), \\
& \left\langle 0|\eta(0)| \frac{1}{2}^{-}\right\rangle=\lambda^{-} \gamma_{5} v(p),
\end{aligned}
$$

with $\left|\frac{1}{2}^{ \pm}\right\rangle=\left|J^{P}\right\rangle$, and $v(p)$ is the Dirac spinor normalized such that $\bar{v}(p) v(p)=2 m$.

In order to evaluate the correlation function $\Pi(p)$ at the quark level, we first need to determine the quark propagator in the presence of quark and gluon condensates. Keeping track of terms in masses of light quarks and taking into account quark and gluon condensates, we obtain the quark propagator as

$$
\begin{aligned}
i S^{a b} \equiv & \left\langle 0\left|T\left[q^{a}(x) \bar{q}^{b}(0)\right]\right| 0\right\rangle \\
= & \frac{i \delta^{a b}}{2 \pi^{2} x^{4}} \hat{x}+\frac{i}{32 \pi^{2}} \frac{\lambda_{a b}^{n}}{2} g_{c} G_{\mu \nu}^{n} \frac{1}{x^{2}}\left(\sigma^{\mu v} \hat{x}+\hat{x} \sigma^{\mu v}\right)-\frac{\delta^{a b}}{12}\langle\bar{q} q\rangle \\
& +\frac{\delta^{a b} x^{2}}{192}\left\langle g_{c} \bar{q} \sigma G q\right\rangle-\frac{i g_{c}^{2}\langle\bar{q} q\rangle^{2} x^{2}}{2^{5} \times 3^{5}} \delta^{a b} \hat{x}-\frac{g_{c}^{2}\langle\bar{q} q\rangle\left\langle G^{2}\right\rangle x^{4}}{2^{9} \times 3^{3}} \delta^{a b} \\
& -\frac{m_{q} \delta^{a b}}{4 \pi^{2} x^{2}}+\frac{m_{q}}{32 \pi^{2}} \lambda_{a b}^{n} g_{c} G_{\mu \nu}^{n} \sigma^{\mu v} \ln \left(-x^{2}\right)-\frac{\delta^{a b}\left\langle g_{c}^{2} G^{2}\right\rangle}{2^{9} \times 3 \pi^{2}} m_{q} x^{2} \ln \left(-x^{2}\right) \\
& +\frac{i \delta^{a b} m_{q}\langle\bar{q} q\rangle}{48} \hat{x}-\frac{i m_{q}\left\langle g_{c} \bar{q} \sigma G q\right\rangle \delta^{a b} x^{2} \hat{x}}{2^{7} \times 3^{2}}-\frac{g_{c}^{2} m_{q}\langle\bar{q} q\rangle^{2} x^{4} \delta^{a b}}{2^{7} \times 3^{5}} .
\end{aligned}
$$

Here we have adopted $\left\langle g_{c} \bar{q} \sigma G q\right\rangle \equiv\left\langle 0\left|: g_{c} \bar{q} \sigma G q:\right| 0\right\rangle,\langle\bar{q} q\rangle \equiv\left\langle 0\left|: \bar{q}^{a} q^{a}:\right| 0\right\rangle$, and $\hat{x} \equiv \gamma_{\mu} x^{\mu}$. The various terms in Eq. (4) are represented pictorially in Fig. 1 - the first term as Fig. 1(a), the second as Fig. 1(b), and so on. Note that terms proportional to the quark mass $m_{q}$ have not been included consistently in the literature. Note also that the fixed-point gauge [9], $x^{\mu} A_{\mu}(x)=0$, is used to ensure the gauge-invariant nature of the results. (See Appendix B.)

Substituting Eq. (2) into Eq. (1), we obtain

$$
\langle 0|T[\eta(x) \bar{\eta}(0)]| 0\rangle=i 2 \epsilon^{a b c} \epsilon^{a^{\prime} b^{\prime} c^{\prime}} \operatorname{Tr}\left\{S_{u}^{b b^{\prime}}(x) \gamma_{v} C\left[S_{u}^{a a^{\prime}}(x)\right]^{T} C \gamma_{\mu}\right\} \gamma_{5} \gamma^{\mu} S_{d}^{c c^{\prime}}(x) \gamma^{v} \gamma_{5}
$$

Subsequently, the substitution of Eq. (4) into Eq. (5) results in the nonvanishing contributions which are represented pictorially as Figs. $2(\mathrm{a})-2(\mathrm{~m})$. The explicit final results on $\langle 0|T(\eta(x) \bar{\eta}(0))| 0\rangle$ and $\Pi(p)$ are listed immediately below:

$-\frac{24 i \hat{x}}{\pi^{6} x^{10}}$$$
-\frac{i\left\langle g_{c}^{2} G^{2}\right\rangle \hat{x}}{32 \pi^{6} x^{6}}
$$$$
\frac{i\langle\bar{u} u\rangle^{2} \hat{x}}{3 \pi^{2} x^{4}}
$$$$
-\frac{i\langle\bar{u} u\rangle\left\langle g_{c} \bar{u} \sigma G u\right\rangle \hat{x}}{48 \pi^{2} x^{2}}
$$

$$
\frac{2\langle\bar{d} d\rangle}{\pi^{4} x^{6}}
$$

0

$$
-\frac{\left\langle g_{c}^{2} G^{2}\right\rangle\langle\bar{d} d\rangle}{2^{5} \times 3^{2} \pi^{4} x^{2}}
$$$$
\frac{17 g_{c}^{2}\langle\bar{u} u\rangle^{2}\langle\bar{d} d\rangle \ln \left(-x^{2}\right)}{2 \times 3^{4} \pi^{2}}
$$

$$
\begin{aligned}
& -\frac{p^{4}\left[\ln \left(-p^{2}\right)+\frac{1}{3}\right]}{4(2 \pi)^{4}} \hat{p} \\
& -\frac{\left[\ln \left(-p^{2}\right)+1\right]\left\langle g_{c}^{2} G^{2}\right\rangle}{8(2 \pi)^{4}} \hat{p} \\
& -\frac{2\langle\bar{u} u\rangle^{2}}{3 p^{2}} \hat{p} \\
& \frac{\langle\bar{u} u\rangle\left\langle g_{c} \bar{u} \sigma G u\right\rangle}{6 p^{4}} \hat{p} \\
& \frac{p^{2} \ln \left(-p^{2}\right)\langle\bar{d} d\rangle}{4 \pi^{2}}
\end{aligned}
$$

0

$$
\begin{aligned}
& -\frac{\left\langle g_{c}^{2} G^{2}\right\rangle\langle\bar{d} d\rangle}{2^{3} \times 3^{2} \pi^{2} p^{2}} \\
& -\frac{136 g_{c}^{2}\langle\bar{u} u\rangle^{2}\langle\bar{d} d\rangle}{3^{4} q^{4}}
\end{aligned}
$$


$2(\mathrm{~m})$

$$
-\frac{i m_{d}\langle\bar{d} d\rangle \hat{x}}{\pi^{4} x^{6}}
$$$$
-\frac{i m_{d} a_{d} m_{0}^{2} \hat{x}}{192 \pi^{6} x^{4}}-\frac{i m_{u} a_{u} m_{0}^{2} \hat{x}}{96 \pi^{6} x^{4}}
$$$$
\frac{6 m_{d}}{\pi^{6} x^{8}}
$$$$
-\frac{m_{d}\left\langle g_{c}^{2} G^{2}\right\rangle}{2^{7} \pi^{6} x^{4}}
$$$$
-\frac{m_{d}\langle\bar{u} u\rangle^{2}}{3 \pi^{2} x^{2}}-\frac{m_{u}\langle\bar{u} u\rangle\langle\bar{d} d\rangle}{2 \pi^{2} x^{2}}
$$

$$
\begin{aligned}
& -\frac{m_{d}\langle\bar{d} d\rangle}{4 \pi^{2}}\left[\ln \left(-p^{2}\right)+1\right] \hat{p} \\
& +\frac{m_{d} a_{d} m_{0}^{2}}{96 \pi^{4} p^{2}} \hat{p}+\frac{m_{u} a_{u} m_{0}^{2}}{48 \pi^{4} p^{2}} \hat{p} \\
& -\frac{m_{d}}{2^{5} \pi^{4}} p^{4} \ln \left(-p^{2}\right) \\
& \frac{m_{d}\left\langle g_{c}^{2} G^{2}\right\rangle \ln \left(-p^{2}\right)}{2^{7} \pi^{4}} \\
& -\frac{4 m_{d}\langle\bar{u} u\rangle^{2}}{3 p^{2}}-\frac{2 m_{u}\langle\bar{u} u\rangle\langle\bar{d} d\rangle}{p^{2}}
\end{aligned}
$$

To determine the correlation function at the hadron level, we use the dispersion relation

$$
\Pi(p)=\frac{1}{\pi} \int_{0}^{\infty} \frac{\operatorname{Im} \Pi\left(p^{\prime}\right)}{p^{\prime 2}-p^{2}-i \varepsilon} d p^{\prime 2},
$$

where

$$
\operatorname{Im} \Pi\left(p^{\prime}\right)=\pi \sum_{X} \delta\left(p^{\prime 2}-M_{X}^{2}\right)\langle 0|\eta| X\rangle\langle X|\bar{\eta}| 0\rangle .
$$

Here the parity of the intermediate state $X$ can either be positive or negative.

The QCD sum rules for the proton mass may be obtained by comparing the coefficients of the terms proportional to $\hat{p}$ and 1. To accentuate the contributions from the ground state (the nucleon), we apply a Borel transform to both the results obtained at the quark-gluon level and at the hadron level. (See Appendix A.) The resultant QCD sum rules for the proton mass are given by

$$
\frac{M^{6}}{8}+\frac{M^{2} b}{32}+\frac{a_{u}^{2}}{6}-\frac{a_{u}^{2} m_{0}^{2}}{24 M^{2}}-\frac{m_{d} a_{d} M^{2}}{4}-\frac{m_{d} a_{d} m_{0}^{2}}{24}-\frac{m_{u} a_{u} m_{0}^{2}}{12}=\beta_{p}^{2} e^{-\left(M_{p}^{2} / M^{2}\right)}+\text { excited states }+ \text { the continuum, }
$$

and

$\frac{a_{d} M^{4}}{4}-\frac{a_{d} b}{72}+\frac{34}{81} \frac{\alpha_{s}}{\pi} \frac{a_{u}^{2} a_{d}}{M^{2}}+\frac{m_{d} M^{6}}{4}-\frac{m_{d} b M^{2}}{32}+\frac{m_{d} a_{u}^{2}}{3}+\frac{m_{u} a_{u} a_{d}}{2}$

where

$$
=\beta_{p}^{2} M_{p} e^{-\left(M_{p}^{2} / M^{2}\right)}+\text { excited states }+ \text { the continuum },
$$

$$
\begin{aligned}
& a_{q} \equiv-(2 \pi)^{2}\langle\bar{q} q\rangle, \\
& b \equiv\left\langle g_{c}^{2} G^{2}\right\rangle, \\
& a_{q} m_{0}^{2} \equiv(2 \pi)^{2}\left\langle g_{c} \bar{q} \sigma \cdot G q\right\rangle, \\
& \beta_{N}^{2} \equiv(2 \pi)^{4} \frac{\lambda_{N}^{2}}{4} .
\end{aligned}
$$

Our results differ from previous ones primarily in terms proportional to quark masses. In addition, note that, in Eq. (9), the coefficient of $a_{d} b$ differs somewhat from that reported earlier in Ref. [10]; the difference results in only a marginally important numerical difference. Note also that only the QCD sum rule (8) with $m_{d}=m_{u}=0$ (the Belyaev-Ioffe mass rule [4]) has been used extensively in the literature.

Exchanging $u$ and $d$, we may write down immediately the QCD sum rules for the neutron mass:

$$
\frac{M^{6}}{8}+\frac{M^{2} b}{32}+\frac{a_{d}^{2}}{6}-\frac{a_{d}^{2} m_{0}^{2}}{24 M^{2}}-\frac{m_{u} a_{u} M^{2}}{4}-\frac{m_{u} a_{u} m_{0}^{2}}{24}-\frac{m_{d} a_{d} m_{0}^{2}}{12}=\beta_{n}^{2} e^{-\left(M_{n}^{2} / M^{2}\right)}+\text { excited states }+ \text { the continuum , }
$$

and

$$
\begin{aligned}
\frac{a_{u} M^{4}}{4}-\frac{a_{u} b}{72}+\frac{34}{81} \frac{\alpha_{s}}{\pi} \frac{a_{d}^{2} a_{u}}{M^{2}}+\frac{m_{u} M^{6}}{4}-\frac{m_{u} b M^{2}}{32}+\frac{m_{u} a_{d}^{2}}{3} & +\frac{m_{d} a_{d} a_{u}}{2} \\
& =\beta_{n}^{2} M_{n} e^{-\left(M_{n}^{2} / M^{2}\right)}+\text { excited states + the continuum }
\end{aligned}
$$




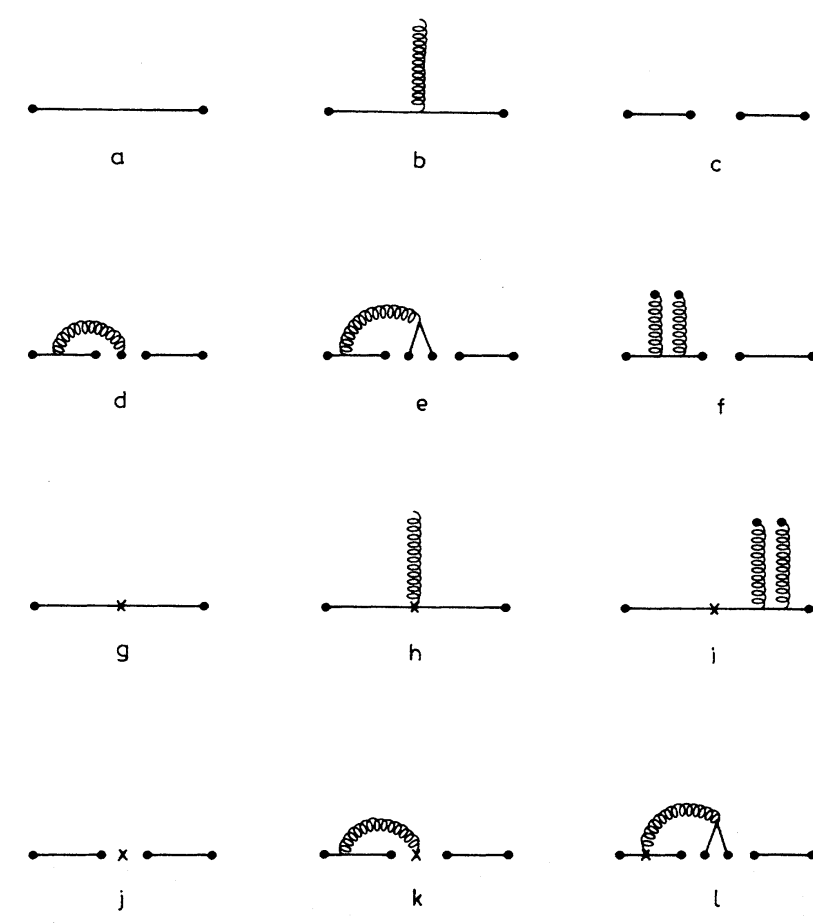

FIG. 1. Diagrams for the propagator of Eq. (4).
According to Eq. (6), we may describe contributions from the nucleon, excited states, and the continuum approximately as $[1,2,7]$

$$
\begin{aligned}
\operatorname{Im} \Pi\left(p^{\prime}\right)= & \pi \lambda_{N}^{2}\left(\hat{p}^{\prime}+M_{N}\right) \delta\left(p^{\prime 2}-M_{N}^{2}\right) \\
& +\pi \theta\left(p^{\prime 2}-W^{2}\right) \hat{p}^{\prime} \operatorname{Im} \Pi_{1}\left(p^{\prime 2}\right) \\
& +\pi \theta\left(p^{\prime 2}-W^{2}\right) \operatorname{Im} \Pi_{2}\left(p^{\prime 2}\right)
\end{aligned}
$$

where $W$ is the threshold value of the continuum (and excited states). Note that $W_{n}^{2}$ could differ slightly from $W_{p}^{2}$, giving rise to some uncertainty in the predicted neutronproton mass difference. To proceed further, we may approximate the spectral functions $\operatorname{Im}_{1,2}\left(p^{\prime}\right)$ by those obtained at the quark level. Thus, we write, as an approximation to Eq. (7),

$$
\operatorname{Im} \Pi_{1}\left(p^{\prime 2}\right)=\frac{1}{4(2 \pi)^{4}} p^{\prime 4}+\frac{1}{(2 \pi)^{4}}\left(\frac{b}{8}-m_{d} a_{d}\right),
$$

and

$$
\operatorname{Im} \Pi_{2}\left(p^{\prime 2}\right)=\frac{m_{d}}{2(2 \pi)^{4}} p^{\prime 4}+\frac{a_{d}}{(2 \pi)^{4}} p^{\prime 2}-\frac{m_{d} b}{8(2 \pi)^{4}} .
$$

To improve further the $Q^{2}$ range of the validity of the derived QCD sum rules, it is useful to incorporate the

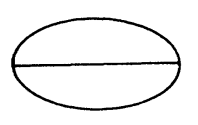

a

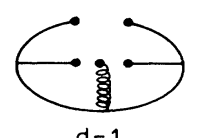

d-1
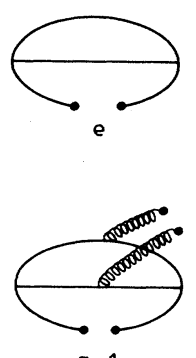

$g-1$
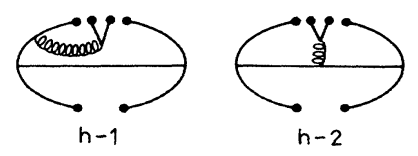

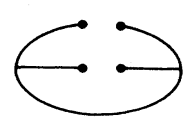

c
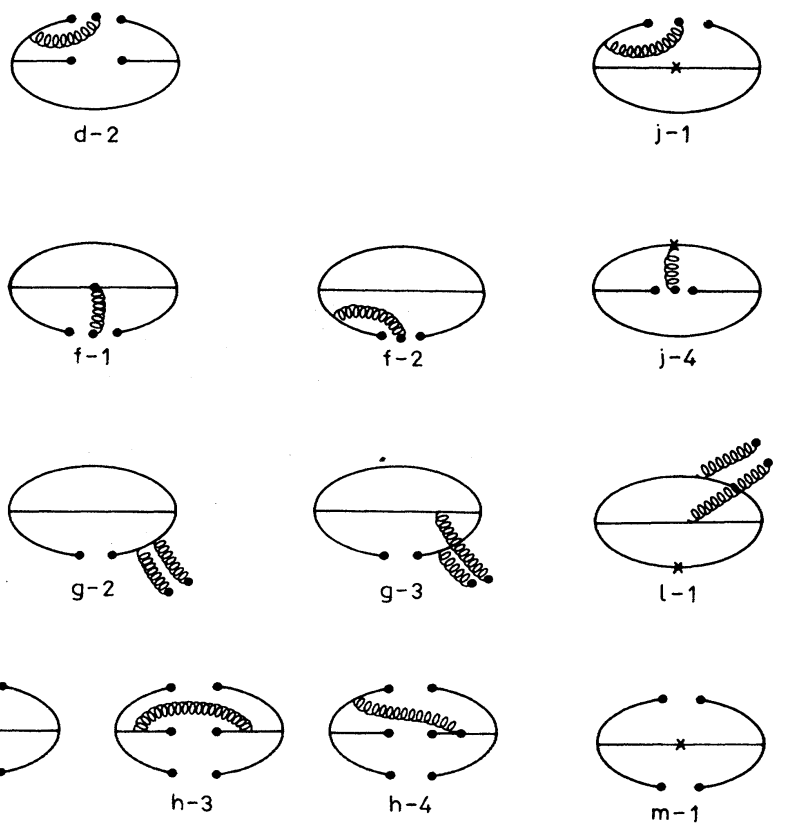
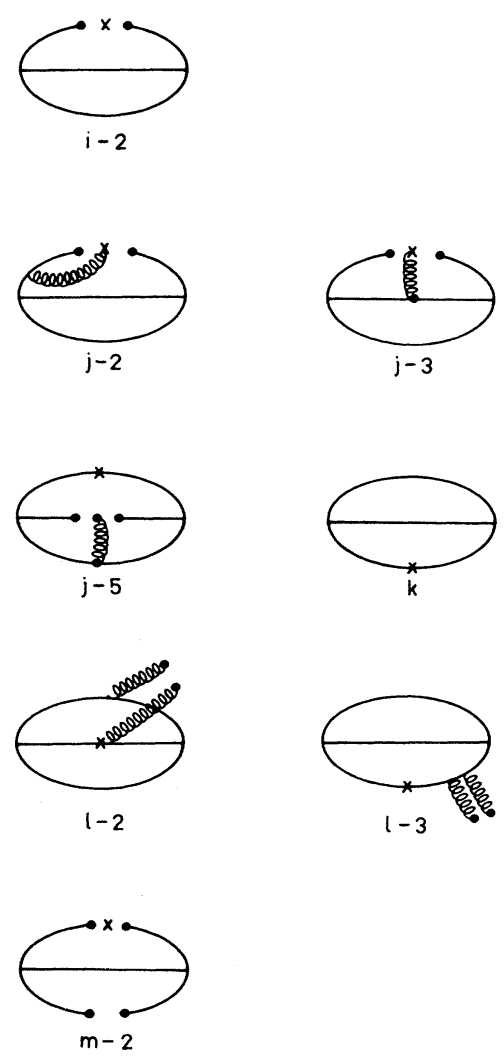

FIG. 2. Diagrams for the two-point correlation function of Eq. (5). 
$Q^{2}$ dependence of the various terms using the renormalization-group ( $R G)$ equation. In particular, it is useful to multiply each term in the operator product expansion by a coefficient $\left\{\left[\ln \left(M^{2} / \Lambda^{2}\right)\right] /\left[\ln \left(\mu^{2} / \Lambda^{2}\right)\right]\right\}^{-2 \gamma_{\eta}+\gamma_{n}}$, where $\mu$ is the renormalization point taken to be $0.5 \mathrm{GeV}, \Lambda$ is the QCD scale parameter taken to be $0.1 \mathrm{GeV}, \gamma_{\eta}$ is the anomalous dimension of the current $\eta$, and $\gamma_{n}$ is the anomalous dimension of the operator under consideration $O_{n}$. (Note that after the Borel transformation, the dependence on $Q^{2}$ is translated into a dependence on the Borel mass, $M$.) Here the anomalous dimensions relevant in our calculation are listed below $[11,12,1]$ :

$$
\begin{aligned}
& \eta(x): \frac{2}{9} ; \\
& \bar{q} q: \frac{4}{9} ; \\
& \alpha_{s} G_{\mu \nu}^{n} G^{n \mu \nu}: 0 ; \\
& m_{q}:-\frac{4}{9} ; \\
& g_{c} \bar{q} \sigma G q: \approx-\frac{2}{27} .
\end{aligned}
$$

Taking into account the aspects related to Eqs. (13) - (15), we finally obtain the following QCD sum rules for the proton mass:

$$
\begin{aligned}
\frac{M^{6}}{8} L^{-4 / 9} E_{2}+\frac{b M^{2}}{32} L^{-4 / 9} E_{0}+\frac{a_{u}^{2}}{6} L^{4 / 9}-\frac{a_{u}^{2} m_{0}^{2}}{24 M^{2}} L^{-2 / 27} & \\
& -\frac{m_{d} a_{d} M^{2}}{4} L^{-4 / 9} E_{0}-\frac{m_{d} a_{d} m_{0}^{2}}{24} L^{-26 / 27}-\frac{m_{u} a_{u} m_{0}^{2}}{12} L^{-26 / 27}=\beta_{p}^{2} e^{-\left(M_{p}^{2} / M^{2}\right)},
\end{aligned}
$$

$\frac{a_{d} M^{4}}{4} E_{1}-\frac{a_{d} b}{72}+\frac{34\left(\alpha_{s} / \pi\right) a_{u}^{2} a_{d}}{81 M^{2}} L^{-1 / 9}+\frac{m_{d} M^{6}}{4} L^{-8 / 9} E_{2}$

$$
-\frac{m_{d} b M^{2}}{32} L^{-8 / 9} E_{0}+\frac{m_{d} a_{u}^{2}}{3}+\frac{m_{u} a_{u} a_{d}}{2}=\beta_{p}^{2} M_{p} e^{-\left(M_{p}^{2} / M^{2}\right)},
$$

where $E_{0}=1-e^{-x}, E_{1}=1-(1+x) e^{-x}$, and $E_{2}=1-\left(1+x+\frac{1}{2} x^{2}\right) e^{-x}$, with $x=W^{2} / M^{2}$. In Eqs. (16) and (17), we have dropped those continuum contributions which are not included in $E_{i}$. The factor $L$ is specified by

$$
L \equiv \frac{\ln \left(M^{2} / \Lambda^{2}\right)}{\ln \left(\mu^{2} / \Lambda^{2}\right)}
$$

Using a simple substitution

$$
\left[\begin{array}{l}
a_{d} \\
a_{u} \\
m_{d} \\
m_{u}
\end{array}\right) \Longleftrightarrow\left[\begin{array}{l}
a_{u} \\
a_{d} \\
m_{u} \\
m_{d}
\end{array}\right),
$$

we obtain the corresponding QCD sum rules for the neutron mass:

$$
\begin{aligned}
& \frac{M^{6}}{8} L^{-4 / 9} E_{2}+\frac{b M^{2}}{32} L^{-4 / 9} E_{0}+\frac{a_{d}^{2}}{6} L^{4 / 9}-\frac{a_{d}^{2} m_{0}^{2}}{24 M^{2}} L^{-2 / 27} \\
& -\frac{m_{u} a_{u} M^{2}}{4} L^{-4 / 9} E_{0}-\frac{m_{u} a_{u} m_{0}^{2}}{24} L^{-26 / 27}-\frac{m_{d} a_{d} m_{0}^{2}}{12} L^{-26 / 27}=\beta_{n}^{2} e^{-\left(M_{n}^{2} / M^{2}\right)}, \\
& \frac{a_{u} M^{4}}{4} E_{1}-\frac{a_{u} b}{72}+\frac{34\left(\alpha_{s} / \pi\right) a_{d}^{2} a_{u}}{81 M^{2}} L^{-1 / 9}+\frac{m_{u} M^{6}}{4} L^{-8 / 9} E_{2}-\frac{m_{u} b M^{2}}{32} L^{-8 / 9} E_{0}+\frac{m_{u} a_{d}^{2}}{3}+\frac{m_{d} a_{d} a_{u}}{2}=\beta_{n}^{2} M_{n} e^{-\left(M_{n}^{2} / M^{2}\right)}
\end{aligned}
$$

Note that we have taken into account the possible difference between $\beta_{p}$ and $\beta_{n}$ as induced by $m_{d} \neq m_{u}$ and $a_{d} \neq a_{u}$ (i.e., $\left.\langle 0|: \bar{u} u:| 0\rangle \neq\langle 0|: \bar{d} d:| 0\rangle\right)$. The "explicit" electromagnetic contributions, as illustrated by Figs. $3(a)-3(c)$, are to be included in our numerical analysis. Regularization is needed for a quantitative treatment of the contributions from Fig. 3(a); by inspection, we insert into Eqs. (16) and (17) and (20) and (21) separately the corrections $\frac{4}{9} \chi \frac{1}{8} M^{6} L^{-4 / 9} E_{2}$ and $\frac{1}{9} \chi \frac{1}{8} M^{6} L^{-4 / 9} E_{2}$ and, without the tedious calculation (yet to be formulated), choose to treat $\chi$ as a parameter (characterizing small electromagnetic corrections typically of less than one percent of the total). Contributions from Figs. 3(b), 3(c-1), and $3(\mathrm{c}-2)$ can easily be calculated; only a net correction to Eq. (17) is found and Eqs. (16), (20), and (21) are not affected. Altogether, we find that inclusion of the explicit electromagnetic effect is essential for attaining the overall consistency of the numerical results.

Note also that, in writing down Eqs. (20) and (21), we have assumed $W_{n}^{2}=W_{p}^{2}$ for the sake of illustration. This 

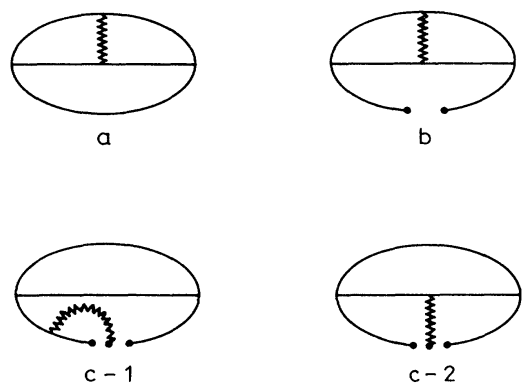

FIG. 3. Diagrams representing the "explicit" electromagnetic corrections to the proton and neutron mass sum rules.

assumption will be discussed later in the next section, as we recognize that the slight difference between $W_{n}^{2}$ and $W_{p}^{2}$ may be the major source for the theoretical error on the predicted neutron-proton mass difference.

\section{NUMERICAL ANALYSIS AND SAMPLE RESULTS}

Different methods may be adopted in the numerical analysis of the QCD sum rules; they yield predictions which may not be consistent among themselves. This feature occurs because not all sum rules are equally good. To establish the viability of the method of QCD sum rules, it is essential to explore different methods adopted in the numerical analysis. To this end, we may view Eqs. (16), (17), (20), and (21) as the "primary" QCD sum rules and those which follow from the primary ones as the "derived" sum rules. In addition, the two QCD sum rules for the proton mass, Eqs. (16) and (17), do not necessarily give rise to completely consistent results, since corrections to the Lorentz structures $\hat{p}$ and 1 may in principle differ from each other. Thus, at first we shall analyze separately these two sum rules, and only at the very end follow Hatsuda, Hogaasen, and Prakash [5] in combining the two sum rules in order to get rid of the factor $\beta_{N}^{2}$.

In our first analysis, we take the logarithm of both sides of Eq. (16) and apply the differential operator $M^{4} \partial / \partial M^{2}$ to both sides. This procedure was used previously by Ioffe and Smilga [10]; it has the advantage of treating efficiently a single sum rule (i.e., getting rid of the parameter $\beta_{p}^{2}$ without a need to combine two different sum rules - perhaps with slightly different behaviors). In this way, we obtain a sum rule which is free of the parameter $\beta_{p}^{2}$ :

$$
\begin{aligned}
& M^{4} \frac{\partial}{\partial M^{2}} \ln \left(\frac{\left(1+\frac{4}{9} \chi\right) M^{6}}{8} L^{-4 / 9} E_{2}+\frac{b M^{2}}{32} L^{-4 / 9} E_{0}+\frac{a_{u}^{2}}{6} L^{4 / 9}-\frac{a_{u}^{2} m_{0}^{2}}{24 M^{2}} L^{-2 / 27}-\frac{m_{d} a_{d} M^{2}}{4} L^{-4 / 9} E_{0}\right. \\
& \left.-\frac{m_{d} a_{d} m_{0}^{2}}{24} L^{-26 / 27}-\frac{m_{u} a_{u} m_{0}^{2}}{12} L^{-26 / 27}\right]=M_{p}^{2}
\end{aligned}
$$

Here, as indicated earlier, the "explicit" electromagnetic contribution as illustrated by Fig. 3(a) is included through the term $\frac{4}{9} \chi \frac{1}{8} M^{6} L^{-4 / 9} E_{2}$, with $\chi$ treated as a free parameter [to be chosen so as to ensure consistency between the two sum rules, Eq. (22) above and Eq. (23) immediately below]. A different procedure to include the pure electromagnetic effects will be discussed later.

Analogously, we obtain, from Eqs. (17), (20), and (21),

$$
\begin{aligned}
& M^{4} \frac{\partial}{\partial M^{2}} \ln \left[\frac{\left(1+\frac{4}{9} \chi\right) a_{d} M^{4}}{4} E_{1}-\frac{a_{d} b}{72}+\frac{34\left(\alpha_{s} / \pi\right) a_{u}^{2} a_{d}}{81 M^{2}} L^{-1 / 9}+\frac{m_{d} M^{6}}{4} L^{-8 / 9} E_{2}-\frac{m_{d} b M^{2}}{32} L^{-8 / 9} E_{0}\right. \\
& \left.+\frac{m_{d} a_{u}^{2}}{3}+\frac{m_{u} a_{u} a_{d}}{2}+\frac{m_{\mathrm{em}}^{2} a_{q} M^{2} E_{0}}{24}\right]=M_{p}^{2}, \\
& M^{4} \frac{\partial}{\partial M^{2}} \ln \left[\frac{\left(1+\frac{1}{9} \chi\right) M^{6}}{8} L^{-4 / 9} E_{2}+\frac{b M^{2}}{32} L^{-4 / 9} E_{0}+\frac{a_{d}^{2}}{6} L^{4 / 9}-\frac{a_{d}^{2} m_{0}^{2}}{24 M^{2}} L^{-2 / 27}-\frac{m_{u} a_{u} M^{2}}{4} L^{-4 / 9} E_{0}\right. \\
& M^{4} \frac{\partial}{\partial M^{2}} \ln \left[\frac{\left(1+\frac{1}{9} \chi\right) a_{u} M^{4}}{4} E_{1}-\frac{a_{u} b}{72}+\frac{34\left(\alpha_{s} / \pi\right) a_{d}^{2} a_{u}}{81 M^{2}} L^{-1 / 9}+\frac{m_{u} M^{6}}{4} L^{-8 / 9} E_{2}-\frac{m_{u} b M^{2}}{32} L^{-8 / 9} E_{0}\right. \\
& \left.\frac{m_{d} a_{d} m_{0}^{2}}{12} L^{-26 / 27}\right)=M_{n}^{2}, \\
& \left.+\frac{m_{u} a_{d}^{2}}{3}+\frac{m_{d} a_{d} a_{u}}{2}\right]=M_{n}^{2} .
\end{aligned}
$$

In Eq. (23), we have introduced $m_{\mathrm{em}}^{2} a_{q} \equiv(2 \pi)^{2}\langle e \bar{q} \sigma \cdot F q\rangle$, with $\langle\bar{u} \sigma \cdot F u\rangle=\frac{2}{3}\langle\bar{q} \sigma \cdot F q\rangle$ and $\langle\bar{d} \sigma \cdot F d\rangle=-\frac{1}{3}\langle\bar{q} \sigma \cdot F q\rangle$. $\left(F_{\mu \nu}\right.$ is the electromagnetic-field-strength tensor.) To attain consistent fits, we choose $m_{\mathrm{em}}^{2}=0.048 \mathrm{GeV}^{2}$. $\left[m_{\mathrm{em}}^{2} / m_{0}^{2}=\boldsymbol{O}\left(\alpha / \alpha_{s}\right)\right.$; see Fig. 5 below. $]$

To decide the range of the Borel mass in which the QCD sum rules may be safely used, we may set $E_{2}, E_{1}$, and $E_{0}$ to 
unity in Eqs. (16), (17), (20), and (21) and compare the difference numerically. More explicitly, we may introduce, for Eq. (16) or (20),

$$
\delta^{(1)} \equiv \frac{\frac{M^{6}}{8} L^{-4 / 9}\left(1-E_{2}\right)+\frac{b M^{2}}{32} L^{-4 / 9}\left(1-E_{0}\right)-\frac{m_{d} a_{d} M^{2}}{4} L^{-4 / 9}\left(1-E_{0}\right)}{\frac{M^{6}}{8} L^{-4 / 9}+\frac{b M^{2}}{32} L^{-4 / 9}+\frac{a_{u}^{2}}{6} L^{4 / 9}-\frac{a_{u}^{2} m_{0}^{2}}{24 M^{2}} L^{-2 / 27}-\frac{m_{d} a_{d} M^{2}}{4} L^{-4 / 9}-\frac{m_{d} a_{d} m_{0}^{2}}{24} L^{-26 / 27}-\frac{m_{u} a_{u} m_{0}^{2}}{12} L^{-26 / 27}} .
$$

(26a)

Note that the denominator is just the left-hand side of Eq. (16) with $E_{2}, E_{1}$, and $E_{0}$ all set to unity, while the numerator is the difference between the left-hand side of Eq. (16) and the denominator. It is clear that $\delta^{(1)}$ is a gauge for the numerical importance of the contribution from the excited states and the continuum to the left-hand side of Eq. (16) (as estimated in the continuum approximation). Analogously, we introduce, for Eq. (17) or (21),

$$
\delta^{(2)} \equiv \frac{\frac{a_{d} M^{4}}{4}\left(1-E_{1}\right)+\frac{m_{d} M^{6}}{4} L^{-8 / 9}\left(1-E_{2}\right)-\frac{m_{d} b M^{2}}{32}\left(1-E_{0}\right) L^{-8 / 9}}{\frac{a_{d} M^{4}}{4}-\frac{a_{d} b}{72}+\frac{34}{81} \frac{\alpha_{s}}{\pi} \frac{a_{u}^{2} a_{d}}{M^{2}} L^{-1 / 9}+\frac{m_{d} M^{6}}{4} L^{-8 / 9}-\frac{m_{d} b M^{2}}{32} L^{-8 / 9}+\frac{m_{d} a_{u}^{2}}{3}+\frac{m_{u} a_{u} a_{d}}{2}}
$$

In Table I, the values of $\delta^{(1),(2)}$, which reflect directly the contributions from excited states, are listed as functions of the Borel mass squared $\boldsymbol{M}^{2}$. Here we have used $a_{u}=a_{d}=0.546 \mathrm{GeV}^{2}$ and $m_{u}=m_{d}=7 \mathrm{MeV}$. The range of the Borel mass may be fixed by assuming, e.g., that contributions from excited states, as specified by $\delta^{(1),(2)}$, are less than $50 \%$ and that the expansion (operator production expansion) in powers of $M^{-2}$ converges reasonably fast. In this way, we find that $0.9 \mathrm{GeV}^{2}<M^{2}<1.1$ $\mathrm{GeV}^{2}$ is a reasonable choice for Eq. (16) or (20), while 0.8 $\mathrm{GeV}^{2}<M^{2}<1.1 \mathrm{GeV}^{2}$ is for Eq. (17) or (21).

In the limit that $m_{d}=m_{u}$ and $a_{d}=a_{u}$, Eqs. (22) and (24) reduce to a single equation (the first mass sum rule) for the nucleon, while Eqs. (23) and (25) reduce to another equation (the second mass sum rule) for the nucleon. We may then obtain the nucleon mass as a function of the square of the Borel mass $M^{2}$. The results are illustrated in Fig. 4, where the solid curve is obtained from the first mass sum rule, while the dashed curve is obtained from the second mass sum rule. The input parameters in the numerical analysis are taken to be the commonly accepted ones $[4,13,14]$ :

TABLE I. The values of $\delta^{(1),(2)}$, defined by Eqs. (26a) and (26b) in the text, are listed as functions of the Borel mass squared $M^{2} . a_{u}=a_{d}=0.546 \mathrm{GeV}^{2}$ and $m_{u}=m_{d}=7 \mathrm{MeV}$ have been used.

\begin{tabular}{lcc}
\hline \hline$M^{2}\left(\mathrm{GeV}^{2}\right)$ & For $\delta^{(1)}(\%)$ & For $\delta^{(2)}(\%)$ \\
\hline 0.6 & 9.8 & 8.9 \\
0.7 & 16.6 & 14.6 \\
0.8 & 24.2 & 20.8 \\
0.9 & 32.1 & 27.0 \\
1.0 & 39.8 & 32.8 \\
1.1 & 46.8 & 38.2 \\
1.2 & 53.2 & 43.2 \\
\hline \hline
\end{tabular}

$$
\begin{aligned}
& b=0.474 \mathrm{GeV}^{4}, \\
& m_{0}^{2}=0.8 \mathrm{GeV}^{2}, \\
& W_{p, n}^{2}=2.25 \mathrm{GeV}^{2}, \\
& \alpha_{s}(\mu)=\frac{4 \pi}{9 \ln \left(\mu^{2} / \Lambda^{2}\right)}=0.434
\end{aligned}
$$

It is seen from Fig. 4 that the observed nucleon mass (939 $\mathrm{MeV}$ ) is reproduced reasonably well.

To study numerically the neutron-proton mass difference, we adopt $[4,13,14]$

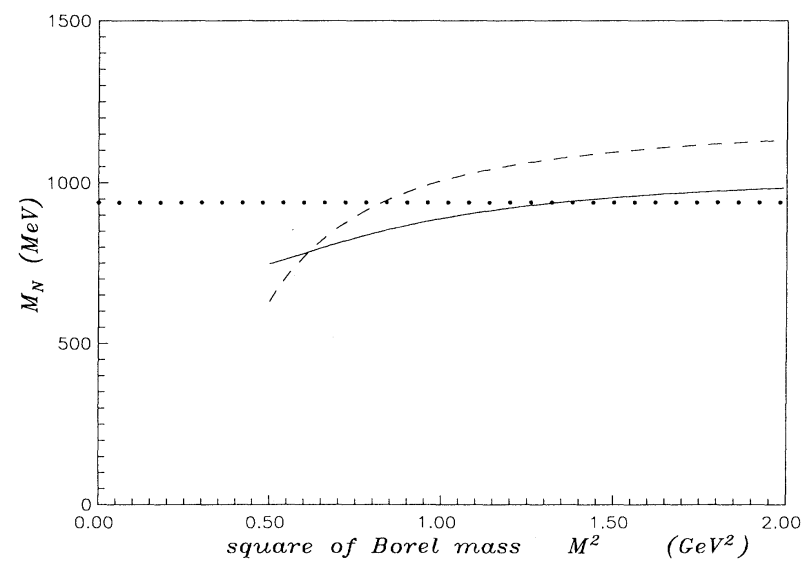

FIG. 4. The nucleon mass as a function of the square of the Borel mass $M^{2}$ [from Eqs. (22) and (23) with $m_{d}=m_{u}$ and $a_{d}=a_{u}$ ]. The solid curve is obtained from the first mass sum rule, while the dashed curve is obtained from the second mass sum rule. 


$$
\begin{aligned}
& a_{q}=\frac{a_{u}+a_{d}}{2}=0.546 \mathrm{GeV}, \\
& \gamma \equiv \frac{\langle\bar{d} d\rangle}{\langle\bar{u} u\rangle}-1=-0.00657, \\
& m_{u}=5.1 \mathrm{MeV} \\
& m_{d}=8.9 \mathrm{MeV} .
\end{aligned}
$$

Here a nonzero $\gamma$ represents another source for isospin symmetry breaking. Our value for $\gamma$ is similar to, but slightly less than, that used by Hatsuda, Hogaasen, and Prakash [5], between -0.0067 and -0.0078 . Gasser and Leutwyler [13] obtain via chiral perturbation theory a formula for $\gamma$ which yields $\gamma \approx-0.0074$. Chernyak and Zhitnitsky [15] obtained another estimate $\gamma \approx-0.009$ via QCD sum rules. Our value for $\gamma$, while close to all these values, is dictated by the overall quality of the solutions to the sum rules (22)-(25). By taking the difference between Eqs. (22) and (24), we obtain a sum rule for $2 M_{N}\left(M_{n}-M_{p}\right)$. In Fig. 5, we plot the resultant mass difference as a function of the Borel mass squared. The dotted curve is the result obtained by assuming $\chi=0$ (i.e., neglecting the explicit electromagnetic effect), while the solid curve is the result obtained by choosing an optimal value $\chi=0.0036$. In the former case, we obtain $M_{n}-M_{p}=1.53 \mathrm{MeV}$, while in the latter $M_{n}-M_{p}=1.35$ $\mathrm{MeV}$, considerably closer to the experimental value of $1.293 \mathrm{MeV}$ (shown as the heavy dotted curve in Fig. 5).

Analogously, we obtain by taking the difference between Eqs. (23) and (25) another sum rule for $2 M_{N}\left(M_{n}-M_{p}\right)$. In Fig. 5, we again plot the resultant mass difference as a function of the Borel mass squared. The dashed curve is the result obtained by assuming $\chi=0$ (i.e., neglecting the explicit electromagnetic effect), while the crossed curve is the result obtained by choosing the same optimal value $\chi=0.0036$. In the former case, we obtain $M_{n}-M_{p}=0.07 \mathrm{MeV}$, while in the latter $M_{n}-M_{p}=1.35 \mathrm{MeV}$, in agreement with that from the solid curve. However, errors on the predictions may be fairly large, as we observe that, for this particular sum rule, the final results depend sensitively on the range of the Borel mass used in the fitting.

It seems from Fig. 5 that the explicit electromagnetic effect modifies the two ( $\hat{p}$ and 1$)$ sum rules very differently - for the $\hat{p}$ sum rule, it contributes to $M_{n}-M_{p}$

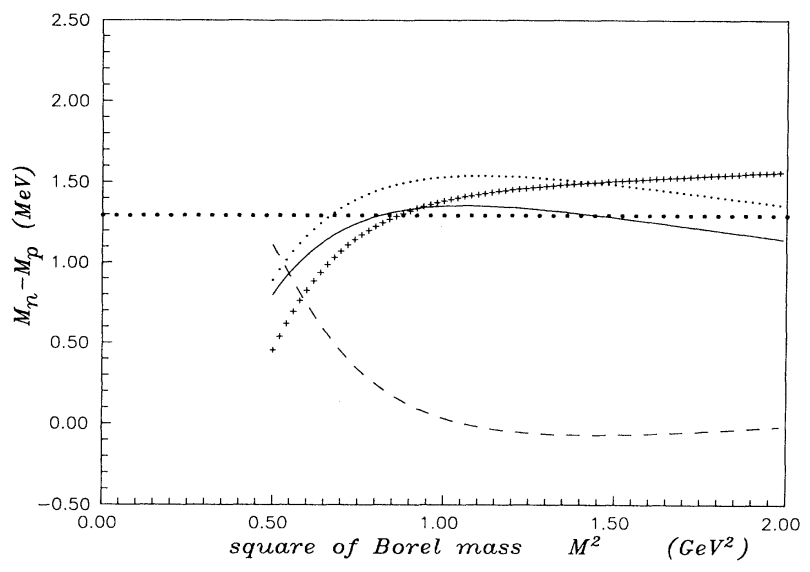

FIG. 5. The neutron-proton mass difference as a function of the Borel mass squared. The dotted curve is the result obtained from taking the difference between Eqs. (22) and (24) and assuming $\chi=0$ (i.e., neglecting the explicit electromagnetic effect), while the solid curve is the result obtained by choosing an optimal value $\chi=0.0036$. The dashed curve is the result obtained from taking the difference between Eqs. (23) and (25) and assuming $\chi=0$, while the crossed curve is the result obtained by choosing the same optimal value $\chi=0.0036$. The experimental value of $1.293 \mathrm{MeV}$ is shown as the heavy dotted curve.

a correction of about $-0.2 \mathrm{MeV}$ (which is quite insensitive to the Borel mass for $M^{2} \geq 0.9 \mathrm{GeV}^{2}$ ), while for the 1 (unity) sum rule the correction increases from a vanishing value at $M^{2} \approx 0.6 \mathrm{GeV}^{2}$ to about $1.3 \mathrm{MeV}$ (the entire observed value for $M_{n}-M_{p}$ ) at $M^{2} \approx 1 \mathrm{GeV}^{2}$. It is clear that the $\hat{p}$ (first) sum rule works well numerically; by comparison, we cannot say much about the unity (second) sum rule. (It is a nontrivial fact that the explicit electromagnetic effect modifies the two sum rules differently - as in quark models, the electromagnetic contribution to the neutron-proton mass difference can be deduced in an almost model-independent manner [16]).

We can take a somewhat different track. We take the difference between Eq. (16) and Eq. (20) and multiply the two sides of the resultant equation by $\left(M^{2} / 2 M_{N} \beta_{N}^{2}\right)$, with $M_{N} \equiv\left(M_{n}+M_{p}\right) / 2$ and $\beta_{N} \equiv\left(\beta_{n}+\beta_{p}\right) / 2$. Noting that $M_{p} \gg\left(M_{n}-M_{p}\right)$ and $\beta_{p} \gg\left|\beta_{n}-\beta_{p}\right|$, we obtain a QCD sum rule for the neutron-proton mass difference:

$$
\begin{array}{r}
{\left[\left(a_{u}^{2}-a_{d}^{2}\right)\left[\frac{L^{4 / 9}}{6}-\frac{m_{0}^{2}}{24 M^{2}} L^{-2 / 27}\right]+\left(m_{u} a_{u}-m_{d} a_{d}\right)\left[\frac{M^{2}}{4} L^{-4 / 9} E_{0}-\frac{m_{0}^{2}}{24} L^{-26 / 27}\right)+\frac{\chi}{24} M^{6} L^{-4 / 9} E_{2}\right] \frac{M^{2}}{2 M_{N} \beta_{N}^{2}}} \\
=\left(\left(M_{n}-M_{p}\right)-\frac{M^{2}}{2 M_{N} \beta_{N}^{2}}\left(\beta_{n}^{2}-\beta_{p}^{2}\right)\right] e^{-\left(M_{N}^{2} / M^{2}\right)}
\end{array}
$$

Analogously, we obtain from Eqs. (17) and (21) another QCD sum rule for the neutron-proton mass difference: 


$$
\begin{aligned}
& {\left[( a _ { d } - a _ { u } ) \left[\frac{M^{4}}{4} E_{1}-\right.\right.}\left.\frac{b}{72}-\frac{34}{81} \frac{\alpha_{s}}{\pi} \frac{a_{d} a_{u}}{M^{2}} L^{-1 / 9}\right]+\left(4 a_{d}-a_{u}\right) \frac{\chi M^{4}}{36} E_{1} \\
&\left.+\left(m_{d}-m_{u}\right)\left[\frac{M^{6}}{4} L^{-8 / 9} E_{2}-\frac{b M^{2}}{32} L^{-8 / 9} E_{0}-\frac{a_{u} a_{d}}{2}\right]+\frac{M_{d} a_{u}^{2}-m_{u} a_{d}^{2}}{3}+\frac{m_{e m}^{2} a_{q} M^{2} E_{0}}{24}\right] \frac{M^{2}}{2 M_{N}^{2} \beta_{N}^{2}} \\
&=\left[\left(M_{n}-M_{p}\right)\left[1-\frac{M^{2}}{2 M_{N}^{2}}\right]-\frac{M^{2}}{2 M_{N} \beta_{N}^{2}}\left(\beta_{n}^{2}-\beta_{p}^{2}\right)\right] e^{-\left(M_{N}^{2} / M^{2}\right)} .(30)
\end{aligned}
$$

Note that we may recast Eqs. (29) and (30) in a linearized version, i.e., to first order in $m_{d}-m_{u}$, or in $a_{d}-a_{u}$, or in $\beta_{n}^{2}-\beta_{p}^{2}$. As anticipated, numerical analyses of linearized sum rules give rise to essentially the same results.

We may use Eq. (29), the first QCD sum rule for the neutron-proton mass difference, to determine $M_{n}-M_{p}$ as a function of the square of the Borel mass, $M^{2}$. Here we perform quadratic fits to both the left-hand $(L)$ and right-hand $(R)$ sides after moving the exponential factor $\exp \left(-M_{N}^{2} / M^{2}\right)$ to the left-hand side. The result is illustrated by Fig. 6, with the solid curve for the left-hand side and the dashed curve for the right-hand side. We obtain $M_{n}-M_{p}=1.42 \mathrm{MeV}$ and $\beta_{n}^{2}-\beta_{p}^{2}=-2.0 \times 10^{-4}$ $\mathrm{GeV}^{6}$ as the best fit for $0.9 \mathrm{GeV}^{2}<M^{2}<1.1 \mathrm{GeV}^{2}$.

Analogously, we illustrate in Fig. 7 numerical results from the analysis of the second QCD sum rules on the neutron-proton mass difference, i.e., Eq. (30). Performing the same analysis as for Eq. (29), we obtain the result as illustrated by Fig. 7, with the solid curve for the left-hand side and the dashed curve for the right-hand side. We obtain $M_{n}-M_{p}=0.95 \mathrm{MeV}$ and $\beta_{n}^{2}-\beta_{p}^{2}=-1.4 \times 10^{-3}$ $\mathrm{GeV}^{6}$ as the best fit for $0.9 \mathrm{GeV}^{2}<M^{2}<1.0 \mathrm{GeV}^{2}$.

We proceed to discuss in some detail possible sources for error in our numerical analyses. As mentioned earlier, it is possible that $W_{n}^{2}$ differs slightly from $W_{p}^{2}$. Such a difference affects $E_{2}, E_{1}$, and $E_{0}$ and distinguishes the threshold of the continuum in the proton and neutron cases. In addition, we note that we should in general replace the right-hand side of Eq. (16), $\beta_{p}^{2} \exp \left(-M_{p}^{2} / M^{2}\right)$, by the expression $\beta_{p}^{2}\left(1+A_{p} M^{2}\right) \exp \left(-M_{p}^{2} / M^{2}\right)$, with $A_{p}$

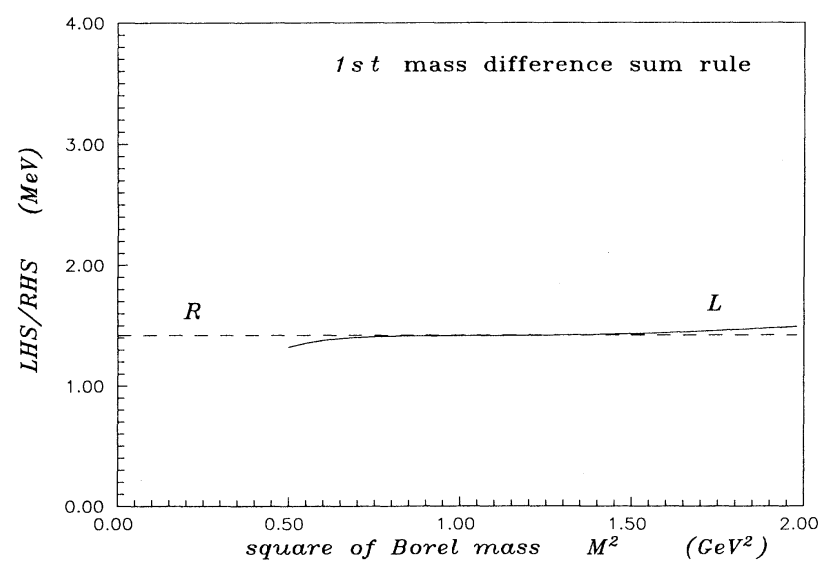

FIG. 6. The neutron-proton mass difference $M_{n}-M_{p}$, as determined from our first QCD sum rule (29), is shown as a function of the square of the Borel mass $M^{2}$. characterizing the "residual" contribution from the excited states or the continuum. Thus, the other source for the difference may come from $A_{p} \neq A_{n}$. Nevertheless, the continuum for the proton may come from a combination of $p \pi^{0}$ and $n \pi^{+}$, while that for the neutron may come from a combination of $n \pi^{0}$ and $p \pi^{-}$. For instance, the first excited $\frac{1}{2}^{+}$state $[N(1440)]$ decays $50-70 \%$ of the time to $N \pi$. Thus, in an average sense, we anticipate $W_{p}^{2}=W_{n}^{2}$ and perhaps $A_{p}=A_{n}$ as well. The effect caused by $A_{p} \neq A_{n}$ is in any event of marginal importance, since most of the effects cancel out when we consider the logarithmic derivatives, as in Eqs. (22)-(25). Thus, we focus on the effect caused by $W_{n}^{2} \neq W_{p}^{2}$; in view of the above argument, it is likely that the difference is in the range of less than $0.1 \%$ [i.e., somewhat smaller than $\left.\left(M_{n}-M_{p}\right) / M_{N}\right]$.

In Fig. 8, we repeat the analyses performed in obtaining Fig. 5 (taking into account the explicit electromagnetic effect as before). For the difference equation from Eqs. (22) and (24), the dotted curve (instead of the previous solid curve in Fig. 5) is obtained if we choose $\left(W_{n}^{2}-W_{p}^{2}\right) / \bar{W}^{2}=+0.1 \%$. This gives rise to $M_{n}-M_{p}=1.84 \mathrm{MeV}$. On the other hand, we obtain the solid curve and $M_{n}-M_{p}=0.87 \mathrm{MeV}$ for $\left(W_{n}^{2}-W_{p}^{2}\right) / \bar{W}^{2}=-0.1 \%$. For the difference equation from Eqs. (23) and (25), we obtain the dashed curve and $M_{n}-M_{p}=1.88 \mathrm{MeV}$ for $\left(W_{n}^{2}-W_{p}^{2}\right) / \bar{W}^{2}=+0.1 \%$ or the crossed curve and $M_{n}-M_{p}=0.89 \mathrm{MeV}$ for $\left(W_{n}^{2}-W_{p}^{2}\right) / \bar{W}^{2}=-0.1 \%$. Thus, assuming that the total

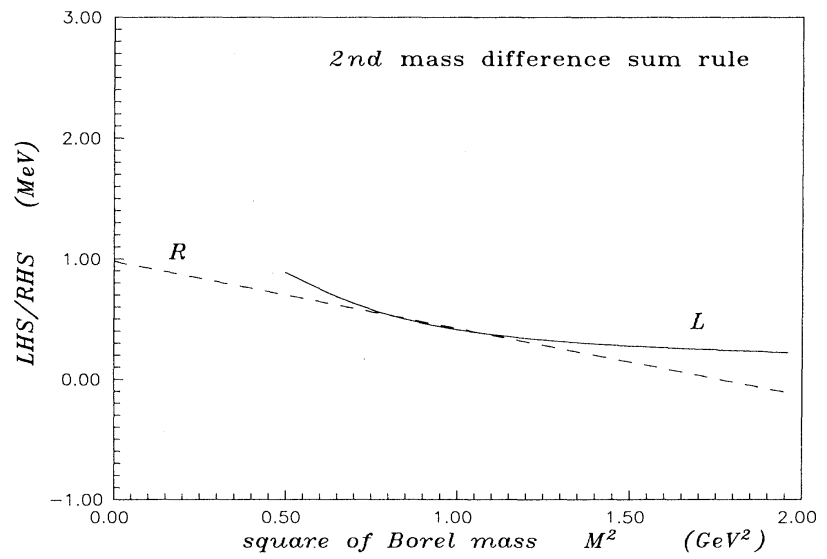

FIG. 7. The neutron-proton mass difference $M_{n}-M_{p}$, as determined from our second QCD sum rule (30), is shown as a function of the square of the Borel mass $\boldsymbol{M}^{2}$. 


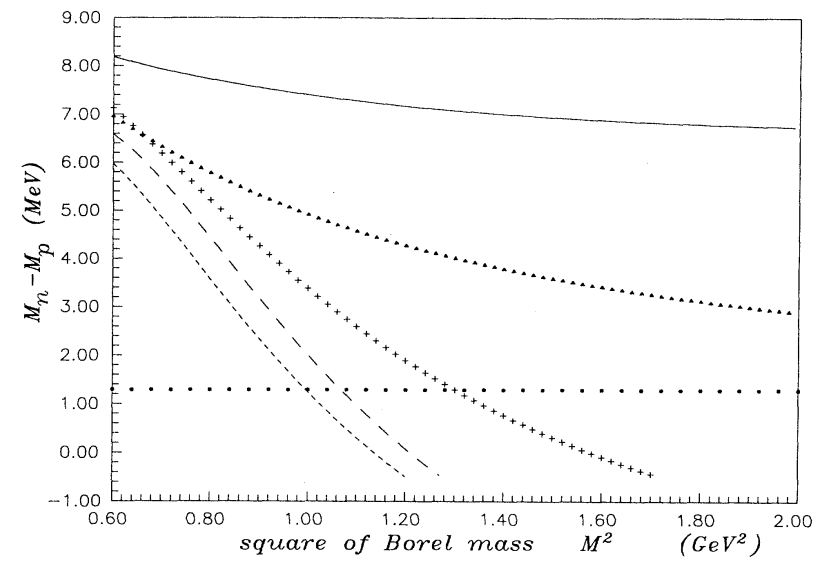

FIG. 8. The error analyses performed for Fig. 5 (taking into account the explicit electromagnetic effect as before). For the difference equation from Eqs. (22) and (24), the dotted curve (instead of the previous solid curve in Fig. 5) is obtained if we choose $\left(W_{n}^{2}-W_{p}^{2}\right) / \bar{W}^{2}=+0.1 \%$. This gives rise to $M_{n}-M_{p}=1.84 \mathrm{MeV}$. On the other hand, we obtain the solid curve and $M_{n}-M_{p}=0.87 \mathrm{MeV}$ for $\left(W_{n}^{2}-W_{p}^{2}\right) / \bar{W}^{2}=-0.1 \%$. For the difference equation from Eqs. (23) and (25), we obtain the dashed curve and $M_{n}-M_{p}=1.88 \mathrm{MeV}$ for $\left(W_{n}^{2}-W_{p}^{2}\right) / \bar{W}^{2}=+0.1 \%$ or the crossed curve and $M_{n}-M_{p}=0.89 \mathrm{MeV}$ for $\left(W_{n}^{2}-W_{p}^{2}\right) / \bar{W}^{2}=-0.1 \%$.

range of uncertainty for $\left(W_{n}^{2}-W_{p}^{2}\right) / \bar{W}^{2}$ is about $0.1 \%$, we have to attribute an error $\pm 0.24 \mathrm{MeV}$ to the (first) $\not p$ sum rule and $\pm 0.25 \mathrm{MeV}$ to the (second) unity sum rule. On the other hand, the analysis of the second sum rule depends fairly sensitively on the range of the Borel mass-we may need to assign an additional error of about $\pm 0.25 \mathrm{MeV}$ to the second sum rule.

We have also performed similar error analyses for Eqs. (29) and (30). Assuming again that the total range of uncertainty for $\left(W_{n}^{2}-W_{p}^{2}\right) / \bar{W}^{2}$ is about $0.1 \%$, we quote the prediction of Eq. (29) as $M_{n}-M_{p}=(1.42 \pm 0.19) \mathrm{MeV}$ and $\beta_{n}^{2}-\beta_{p}^{2}=-(2.0 \pm 0.5) \times 10^{-4} \mathrm{GeV}^{6}$ while that of Eq. (30) as $M_{n}-M_{p}=(0.95 \pm 0.25) \mathrm{MeV}$ and $\beta_{n}^{2}-\beta_{p}^{2}$ $=-(1.4 \pm 0.2) \times 10^{-4} \mathrm{GeV}^{6}$. (To avoid redundance, we shall not present figures which enable us to make these error estimates.)

To sum up, numerical analyses of the difference equation from Eqs. (22) and (24) yield $M_{n}-M_{p}=(1.35 \pm 0.24)$ $\mathrm{MeV}$, consistent with the value of $(1.42 \pm 0.19) \mathrm{MeV}$ as obtained from Eq. (29). Analogously, the difference equation from Eqs. (23) and (25) yields $M_{n}-M_{p}$ $=(1.35 \pm 0.35) \mathrm{MeV}$, consistent with the value of (0.95土0.25) MeV as obtained from Eq. (30).

In concluding this paper, we wish to consider an analysis adopted recently by Hatsuda, Hogaasen, and Prakash [5]. Specifically, Hatsuda, Hogaasen, and Prakash [5] obtained the neutron-proton sum rules using suitable substitutions on the sum rules for $\Sigma$ and $\Xi$. For the proton, they have

$$
\begin{aligned}
& \frac{M^{6}}{8}+\frac{M^{2} b}{32}+\frac{a_{u}^{2}}{6}-\frac{m_{d} a_{d} M^{2}}{4}=\beta_{p}^{2} e^{-\left(M_{p}^{2} / M^{2}\right)} \\
& \frac{a_{d} M^{4}}{4}+\frac{m_{d} M^{6}}{4}+\frac{m_{d} a_{u}^{2}}{3}+\frac{m_{u} a_{u} a_{d}}{2}=\beta_{p}^{2} M_{p} e^{-\left(M_{p}^{2} / M^{2}\right)},
\end{aligned}
$$

which may be contrasted with our Eqs. (16) and (17). Note that several terms (those proportional to $m_{0}^{2}$ and some higher-dimension terms) are absent from Eqs. (31a) and (31b). Note also that, in Eqs. (31a) and (31b), contributions from excited states, as derived from our Eqs. (13) and (14), are neglected, and the $Q^{2}$ behavior due to the anomalous dimension of each term has been left out. Hatsuda, Hogaasen, and Prakash [5] proceed to take the ratio of Eqs. (31b) and (31a) and obtain a sum rule for the proton mass $M_{p}$. Using the substitution Eq. (19), one obtains a similar result for the neutron mass $M_{n}$. Subsequently, one may write down a sum rule for the neutronproton mass difference, to first order in symmetry breaking:

$$
\begin{aligned}
\Delta M=\frac{M_{0}}{a M^{4}}[ & -\left(M^{4}+\frac{4}{3} a M_{0}\right) a \gamma \\
& \left.+\left(\frac{2}{3} a^{2}-M^{6}-a M_{0} M^{2}\right)\left(m_{d}-m_{u}\right)\right],
\end{aligned}
$$

where

$$
a \equiv-(2 \pi)^{2}\langle\bar{q} q\rangle \simeq(2 \pi)^{2}(250 \mathrm{MeV})^{3}
$$

and

$$
\gamma \equiv \frac{\langle\bar{d} d\rangle}{\langle\bar{u} u\rangle}-1 .
$$

$M_{0}$ and $M$ are the nucleon mass in the chiral limit and the Borel mass, respectively. These are determined by $\partial M_{0} / \partial M=0$. Finally, Hatsuda, Hogaasen, and Prakash [5] assume a simple scaling, viz. every mass scale, except for $\left(m_{d}-m_{u}\right)$, is proportional to $\langle\bar{q} q\rangle^{n}$. They obtain

$$
\Delta M=-4.79 \gamma|\langle\bar{q} q\rangle|^{1 / 3}-1.56\left(m_{d}-m_{u}\right) .
$$

We can follow this line of reasoning and obtain a similar sum rule for the neutron-proton mass difference. In Fig. 9, we describe the numerical difference between our sum rule and that of Hatsuda, Hogaasen, and Prakash [5]. The long-dashed curve is the result obtained from the procedure of Hatsuda, Hogaasen, and Prakash [5] together with their parameters: Their condition $\partial M_{N} / \partial M=0$ leads to a nucleon mass $M_{n}=761 \mathrm{MeV}$ at the Borel mass squared $M^{2}=1.044 \mathrm{GeV}^{2}$ and, as a result, one finds $M_{n}-M_{p} \approx 1.5 \mathrm{MeV}$. We would obtain the result shown in the short-dashed curve should we adopt the procedure (formula) of Hatsuda, Hogaasen, and Prakash [5] but with our parameters: $M_{N}=743 \mathrm{MeV}$ and $M_{n}-M_{p} \approx 1.67 \mathrm{MeV}$ at $M^{2}=0.96 \mathrm{GeV}^{2}$. Note that the predicted nucleon mass is in fact a little too low using their procedure.

If we adopt the procedure of Hatsuda, Hogaasen, and Prakash [5] by using our formula together with our pa- 


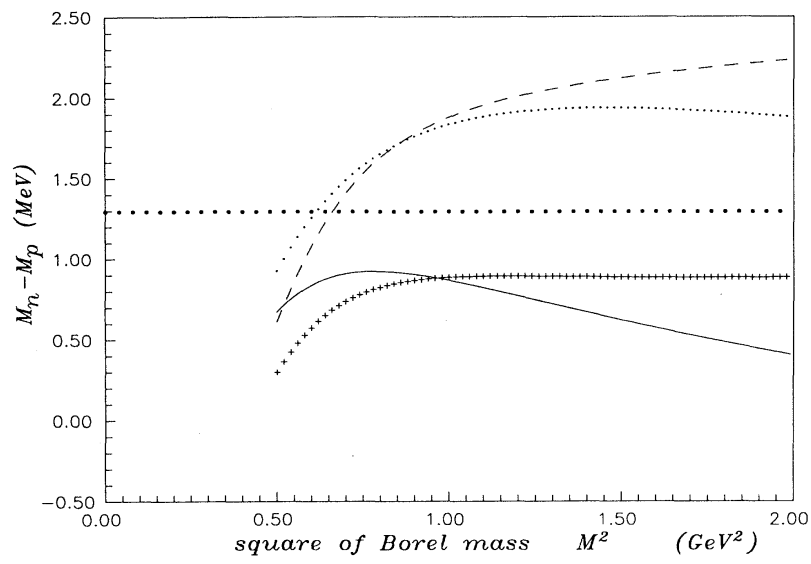

FIG. 9. The numerical difference between our sum rules and that of Hatsuda, Hogaasen, and Prakash [5]: The long-dashed curve is the result obtained from the procedure of Hatsuda, Hogaasen, and Prakash [5] together with their parameters. The result shown in the short-dashed curve is obtained should we adopt Hatsuda's procedure (formula) but with our parameters. If we adopt the procedure of Hatsuda, Hogaasen, and Prakash [5] by using our formula together with our parameters, we obtain the result represented by the solid curve. To understand the difference, we may use our formula and our parameters but with $L=1$ (i.e., neglecting the effect due to anomalous dimensions), yielding the curve marked by triangles. We may also set $W \rightarrow \infty$ (i.e., without proper truncation of the effect due to excited states or continuum), obtaining the curve marked by crosses.

rameters (but without explicit electromagnetic effects), we obtain the result represented by the solid curve in Fig. 9 -an unacceptable result. To understand the differences, we may use our formula and our parameters but with $L=1$ (i.e., neglecting the effect due to anomalous dimensions). The result is shown in the curve marked by triangles. Furthermore, we may also set $W \rightarrow \infty$ (i.e., without proper truncation of the effect due to excited states or continuum) and obtain another curve marked by crosses. It is seen that, if these effects are left out from our formula, we reproduce, step by step, the result of Hatsuda, Hogaasen, and Prakash [5]. The failure may be caused by the fact that the two sum rules, those for $\hat{p}$ and 1 , have different explicit electromagnetic effects and numerically they also behave differently, so that a simple combination of them does not work. These differences make it clear that numerical analyses of QCD sum rules remain a subtle issue.

\section{SUMMARY}

We have used the method of QCD sum rules to investigate the question of the neutron-proton mass difference. We attempt to keep diagrams consistently up to dimension 9, assuming different $u p$ and down current quark masses $\left(m_{u} \neq m_{d}\right)$ and trying to distinguish between $\langle 0|: \bar{u} u:| 0\rangle$ and $\langle 0|: \bar{d} d:| 0\rangle$, the condensates of the $u p$ and down quarks. Using the typical current quark masses, $m_{u}=5.1 \mathrm{MeV}$ and $m_{d}=8.9 \mathrm{MeV}$, and the standard con- densate values, we perform numerical analyses of the resultant QCD sum rules. In particular, numerical analyses of the difference equation from Eqs. (22) and (24) yield $M_{n}-M_{p}=(1.35 \pm 0.24) \mathrm{MeV}$, consistent with the value of (1.42 \pm 0.19$) \mathrm{MeV}$ as obtained from Eq. (29). Analogously, the difference equation from Eqs. (23) and (25) yields $M_{n}-M_{p}=(1.35 \pm 0.35) \mathrm{MeV}$, consistent with the value of $(0.95 \pm 0.25) \mathrm{MeV}$ as obtained from Eq. (30). Altogether, we find that inclusion of the explicit electromagnetic effect is essential for attaining the overall consistency of the numerical results. Our results also suggest that numerical analyses of QCD sum rules remain a subtle issue.

\section{ACKNOWLEDGMENTS}

We would like to thank T. Hatsuda for useful communication. The work of K.-C.Y. and W-Y.P.H. was supported in part by the National Science Council of R.O.C. (NSC82-0208-M002-036). The work of E.M.H. was supported in part by the U.S. Department of Energy, while that of L.S.K. was supported in part by the National Science Foundation. This work was also supported by the N.S.C. of R.O.C. and the National Science Foundation as a cooperative research project.

\section{APPENDIX A: THE BOREL TRANSFORM}

The Borel transform is defined as

$$
\begin{aligned}
B\left[f\left(p^{2}\right)\right]= & \lim _{\substack{n \rightarrow \infty \\
-p^{2} \rightarrow \infty}} \frac{1}{n !}\left(-p^{2}\right)^{n+1}\left(\frac{d}{d p^{2}}\right)^{n} f\left(p^{2}\right) . \\
& \frac{-p^{2}}{M^{2}} \text { fixed }
\end{aligned}
$$

Important examples of Borel transforms include

$$
\begin{aligned}
& B\left[\left(\frac{1}{p^{2}-\alpha}\right]^{\beta}\right]=(-1)^{\beta}(M)^{2-2 \beta} \frac{1}{(\beta-1) !} e^{-\left(\alpha / M^{2}\right)}, \\
& B\left[\left(p^{2}\right)^{m} \ln \left[\frac{1}{-p^{2}}\right]\right]=m !\left(M^{2}\right)^{m+1}, \\
& B\left[\left(p^{2}\right)^{m}\right]=0, m \text { a non-negative integer }, \\
& B\left[f\left(p^{2}\right)\right]=\frac{1}{\pi} \int_{0}^{\infty} d s \operatorname{Im} f(s) e^{-\left(s / M^{2}\right)},
\end{aligned}
$$

if $f\left(p^{2}\right)$ can be written as a dispersion integral;

$$
\begin{aligned}
& B\left[\left(\frac{1}{-p^{2}}\right]^{k}\left[\frac{1}{\ln \left(-p^{2} / \mu^{2}\right)}\right]^{\epsilon}\right] \\
&=\frac{1}{(k-1) !\left(M^{2}\right)^{k-1}}\left[\frac{1}{\ln \left(M^{2} / \mu^{2}\right)}\right]^{\epsilon} \\
& \times\left[1+O\left[\frac{1}{\ln \left(M^{2} / \mu^{2}\right)}\right]\right]
\end{aligned}
$$




\section{APPENDIX B: THE FIXED-POINT GAUGE}

The fixed-point gauge is defined as

$$
x^{\mu} A_{\mu}(x)=0, A_{\mu} \equiv A_{\mu}^{a} \frac{\lambda_{a}}{2} \text {. }
$$

Using

$G_{\mu \nu}=\partial_{\mu} A_{v}-\partial_{v} A_{\mu}+i g\left[A_{\mu}, A_{v}\right], \quad G_{\mu \nu} \equiv G_{\mu \nu}^{a} \frac{\lambda^{a}}{2}$,

multiplying (B2) by $x^{v}$, and using (B1), we get

$$
x^{v} G_{\mu v}=-A_{\mu}-x^{v} \partial_{v} A_{\mu} .
$$

Replacing $x$ by $t x$, we obtain

$$
\frac{d}{d t}\left[t A_{\mu}(t x)\right]=-t x^{v} G_{\mu \nu}(t x)
$$

and

$$
\begin{aligned}
A_{\mu}(x)=-\sum_{k=0}^{\infty} & \frac{1}{k !(k+2)} x^{\nu} x^{\alpha_{1}} x^{\alpha_{2}} \cdots x^{\alpha_{k}} \\
& \times\left(\partial_{\alpha_{1}} \cdots \partial_{\alpha_{k}} G_{\mu \nu}\right)_{x=0} .
\end{aligned}
$$

Note that $A_{\mu}(x=0)=0$ in the fixed-point gauge. We may also recast Eq. (B5) in a gauge-invariant form

$$
\begin{aligned}
A_{\mu}(x)=-\sum_{k=0}^{\infty} & \frac{1}{k !(k+2)} x^{v^{v}} x^{\alpha_{1}} \cdots x^{\alpha_{k}} \\
& \times\left(D_{\alpha_{1}} \cdots D_{\alpha_{k}} G_{\mu v}\right)_{x=0},
\end{aligned}
$$

where $D_{\alpha}=\partial_{\alpha}+i g\left(\lambda^{a} / 2\right) A_{\alpha}^{a}$.
[1] M. A. Shifman, A. I. Vainshtein, and V. I. Zakharov, Nucl. Phys. B147, 385 (1979); B147, 448 (1979).

[2] B. L. Ioffe, Nucl. Phys. B188, 317 (1981); B191, 591(E) (1981).

[3] Y. Chung, H. G. Dosch, M. Kremer, and D. Schall, Nucl. Phys. B197, 55 (1982).

[4] V. M. Belyaev and B. L. Ioffe, Zh. Eksp. Teor. Fiz. 83, 876 (1982) [Sov. Phys. JETP 56, 493 (1982)].

[5] T. Hatsuda, H. Hogaasen, and M. Prakash, Phys. Rev. C 42, 2212 (1990)

[6] L. J. Reinders, H. Rubinstein, and S. Yazaki, Phys. Rep. 127, 1 (1985).

[7] V. M. Belyaev and B. L. Ioffe, Zh. Eksp. Teor. Fiz. 84, 1236 (1983) [Sov. Phys. JETP 57, 716 (1983)].

[8] B. L. Ioffe, Z. Phys. C 18, 67 (1983).

[9] M. A. Shifman, Nucl. Phys. B173, 13 (1980); C. Cronstrom, Phys. Lett. 90B, 267 (1980); M. S. Dubovikov and A. V. Smilga, Nucl. Phys. B185, 109 (1981); J. Schwinger, Particles, Sources, and Fields (Addison-Wesley, New York,
1970).

[10] B. L. Ioffe and A. V. Smilga, Nucl. Phys. B232, 109 (1984).

[11] A. A. Pivovarov and L. R. Surguladze, Nucl. Phys. B360, 97 (1991).

[12] A. I. Vainshtein, V. I. Zakharov, and M. A. Shifman, Pis'ma Zh. Eksp. Teor. Fiz. 23, 656 (1976) [JETP Lett. 23, 602 (1976)]. The anomalous dimension for the operator $\bar{q} \sigma G q$ for three quark flavors is found to be $\approx \frac{23}{54}$ or the anomalous dimension for the operator $g \bar{q} \sigma G q$ is $\approx-\frac{2}{27}$ (which is very close to zero). See also V. M. Belyaev and B. Yu. Blok, Z. Phys. C 30, 279 (1986).

[13] J. Gasser and H. Leutwyler, Phys. Rep. 87, 77 (1982); Nucl. Phys. B250, 465 (1985).

[14] E. M. Henley, W-Y. P. Hwang, and L. S. Kisslinger, Phys. Rev. D 46, 431 (1992).

[15] V. L. Chernyak and A. R. Zhitnitsky, Phys. Rep. 112, 173 (1984).

[16] W-Y. P. Hwang, Phys. Rev. D 31, 2826 (1985); E. M. Henley and G. A. Miller, Nucl. Phys. A518, 207 (1990). 